\title{
Analysis of MIMO-OFDM using different Modulation Techniques
}

\author{
Nidhi Kishor Molgi \\ Department of Electronics Engineering \\ M. S. Bidwe Engineering Collage, \\ Latur
}

\author{
Suresh R. Hallhalli, PhD \\ Department of Electronics Engineering \\ M. S. Bidwe Engineering Collage, \\ Latur
}

\begin{abstract}
MIMO-OFDM system is fast growing new wireless broadband technology which has capability of high data rate transmission and advantages like inter carrier interference (ICI) reduction; high reliability; and better performance in multi-path fading. The major effect to be considered at receiver is fading effects which must be mitigated at receiver using equalization technique. In this paper we demonstrated the BER performance of the OFDM system with two different equalizers ( $\mathrm{ZF}$ and MMSE). Proposed OFDM system is demonstrated using various modulation techniques that is BPSK, QPSK, 16-QAM and 64-QAM using multipath fading channels that is AWGN (Additive White Gaussian Noise) and Rayleigh channel.
\end{abstract}

\section{Keywords}

MIMO, OFDM, BER, ZF and MMSE EQUALIZERS

\section{INTRODUCTION}

In wireless communication technology the main objective is to provide high quality of data. Orthogonal frequency division multiplexing (OFDM) has become a more popular technique for transmission of signals over wireless channels. In OFDM, signals are transmitted in sub channel of different frequency in parallel. The frequency of sub-channel are so selected that these frequencies are orthogonal to each other and therefore do not interfere with each other. This phenomenon makes it possible to transmit the data in overlapping frequency and hence reduces the bandwidth requirement considerably. OFDM is beneficial in many aspects such as high spectral efficiency, robustness, low computational complexity, frequency selective fading, and easy to implementation using IFFT/FFT [1]. In wireless communication systems the data bits are transmitted in radio space, channels are typically multipath fading channels, which causes inter symbol interference (ISI) in the received signal.

ISI is undesirable and it increases bit error rate. ISI causes due to multipath propagation and band limited channels. Whenever the modulation bandwidth exceeds the radio channel coherence bandwidth, ISI is produced. To eliminate ISI from the signal, strong equalizers are used, which requires channel impulse response (CIR) [2]. Equalizer compensate the inter symbol interference means it works in such a way that BER should be low and SNR should be high [3]. Equalization techniques have importance to design of high data rate wireless systems. Most of the wireless receivers are equipped with the equalizer which gives good result. The quality of wireless communication depends upon the three parameters i.e. rate, range and reliability of transmission. These parameters are related with each other. Simultaneous improvement in all three parameters can be accomplished with the help of new technique called MIMO assisted OFDM system. Now a day's integration of OFDM technique with MIMO system has been an area of interesting in the field of broad band wireless communication. MIMO is a frequency selective technique. OFDM can be used to convert such a frequency-selective channel into set of parallel frequency-flat sub channels. MIMO-OFDM system can achieve reliable high data rate transmission over broad band wireless channel [4]. BPSK modulation technique is used in MIMO-OFDM system to evaluate the BER performance. W(n) i.i.d. additive white Gaussian noise sample and $X(n)$ is the discrete time channel impulse response (CIR). At the receiver, firstly serial to parallel conversion occurs and cyclic prefix removed. After removing the $\mathrm{CP}$, the received samples are sent to a fast Fourier transform (FFT) block to de-multiplex the multicarrier signals.

\section{PROPOSED DESIGN}

In this paper, MIMO-OFDM system with an interleaved coded transmission is proposed with different modulation schemes. To demonstrate a coded MIMO-OFDM system is considered with 64 data subcarriers and 16-QAM constellation. Further we also demonstrated system with 128 data subcarriers. To simulate OFDM system Matrix interleaves are used, the cyclic prefix code of length is said to the channel maximum delay, it will prove channel capacity. MIMO-OFDM system increases link reliability, channel capacity and spectral efficiency of multiuser wireless communication [5]. The spectrum signal required to get acceptable result of simulation is first chosen based on the input data and the modulation scheme used (such as Differential BPSK, QPSK or 16-QAM). Amplitudes and phases of the carrier signalsis calculated based on the chosen scheme of modulation. The Transmitting data before transmission is first assigned to each carrier that is to be produced and further it is modulated. The BER performance evaluation of various modulation system such as DPSK, PSK and 16-QAM modulation schemes for $\mathrm{BCH}$ and Convolutionally coded system over AWGN channel is demonstrated in this paper. The performance of different modulation techniques is analyzed in terms of BER and SNR.

To achieve error free communication and multiplexing data, guard interval is inserted in the time domain using cyclic prefix since the ISI gets mitigated between OFDM symbols. The OFDM guard interval can be inserted by the cyclic extensions of the OFDM symbol with cyclic prefix $(\mathrm{CP})$ or cyclic suffix (CS) [9]. In this demonstration we used 16-bit cyclic prefix code. System to be practical, we added AWGN noise to channel. AWGN is a noise that affects the transmitted signal when it passes through the communication channel. It contains a uniform continuous frequency spectrum over a particular frequency band. Here $\mathrm{BCH}$ and Convolutional coding are opted as one knows [7]-[8], This section defines MIMO-OFDM system model as summarized in figure 2, which represents the communication procedure in MIMO-OFDM. Usually fading is seen as a problem in all wireless communication system but MIMO channels uses the 
fading to increase the transmission capacity. The fading effect can be resolved in system with multiple antennas at the transmitter and receiver is known as multiple input multiple output (MIMO) systems. MIMO-OFDM systems transmit different signals from each transmitter element so that the receiving antenna array receives a superposition of all the transmitted signals at receiver. All signals are transmitted from all elements once and the receiver solves a linear equation system to demodulate the message. The multiple antennas are thus used to increase data rates through multiplexing or to improve performance through diversity. MIMO channel model is shown in Fig. 2 with M transmitter and $\mathrm{N}$ receiver antennas. It is achieved by higher spectral efficiency, link reliability and diversity (reduced fading).

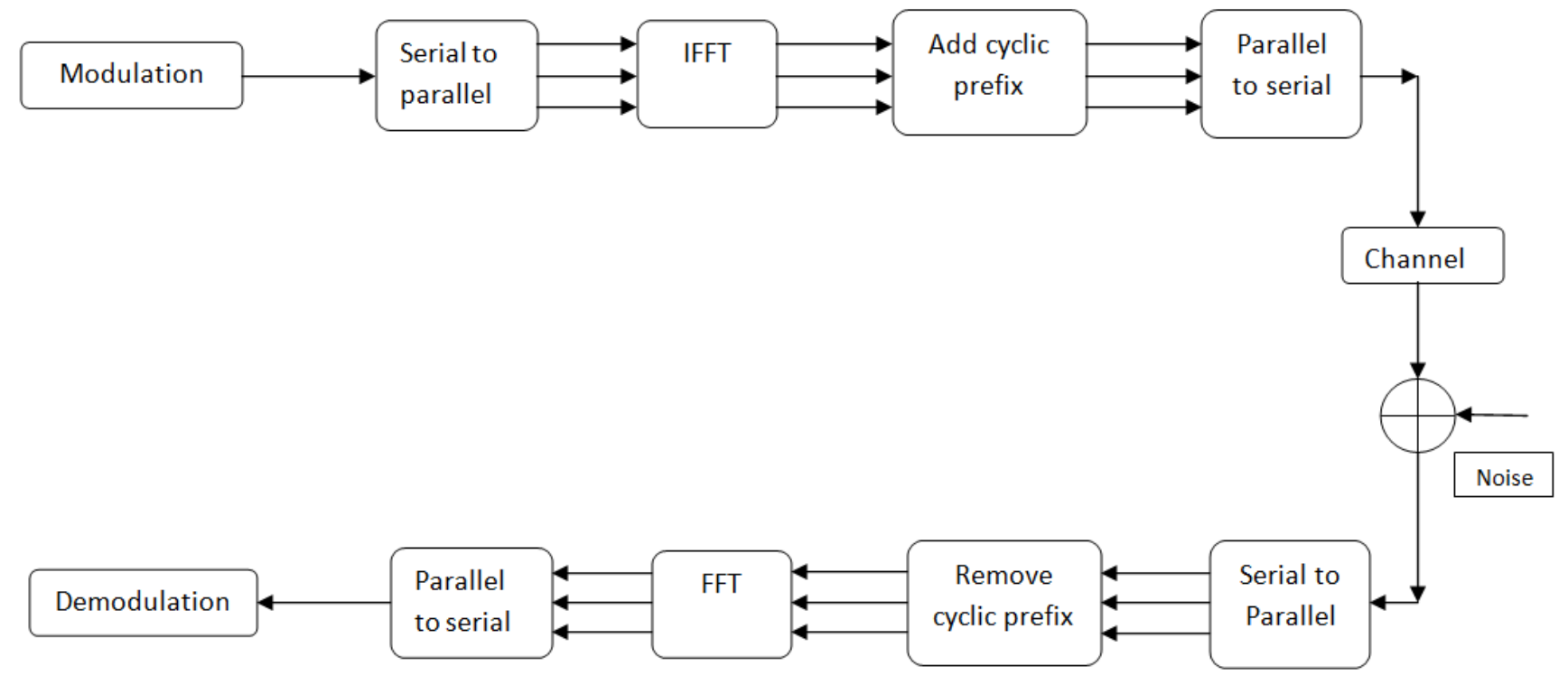

Fig.1. Transmitter and Receiver processing for the generalized OFDM system with Forward Error Correction

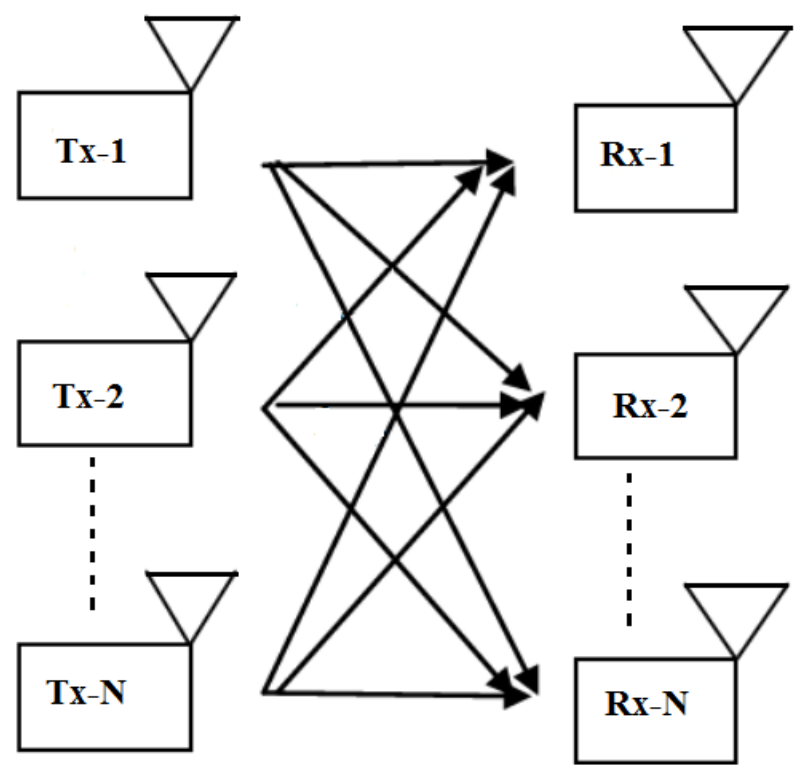

Fig.2 Transmitter and Receiver in MIMO-OFDM system

\section{RESULT AND DISCUSSION}

This paper analyses the bit error rate performance of Convolutionally and $\mathrm{BCH}$ code in AWGN channel using BPSK, QPSK, 16-QAM, 64-QAM as modulation schemes. The $\mathrm{BCH}$ encoder block creates a $\mathrm{BCH}$ code with constraint length $\mathrm{K}=5$. Here a coded OFDM system with 64 subcarriers $(\mathrm{N}=64)$ and the number of constellations for QAM are 16, 64, 256 i.e. 16-QAM, 64-QAM, 256-QAM is considered. Matrix interleaver is used and cyclic prefix is set at $25 \%$ (16). There are two transmit and receive antennas. The figure (4-9) shows the BER Vs SNR for different systems using different modulation schemes. Parameter of proposed system is presented in table 1.OFDM signal with multiple carrier at transmitter by using BPSK modulation is presented in figure 3.

Table 1. Parameters of proposed system

\begin{tabular}{|l|l|}
\hline \multicolumn{1}{|c|}{ Parametrs } & \multicolumn{1}{c|}{ Values } \\
\hline Modulation Techniques & $\begin{array}{l}\text { BPSK, QPSK, 16-QAM, 64- } \\
\text { QAM, 256-QAM }\end{array}$ \\
\hline coding & BCH \\
\hline Interleaver & Matrix \\
\hline Data Subcarriers & 128 \\
\hline Cyclic Prefix & 16 \\
\hline System & OFDM \\
\hline IFFT Points & 128 \\
\hline No. of Samples & 5000 \\
\hline
\end{tabular}

It is even observed from figure that BER keeps improving on using $\mathrm{BCH}$ coding for BPSK, QPSK, and 16-QAM respectively.

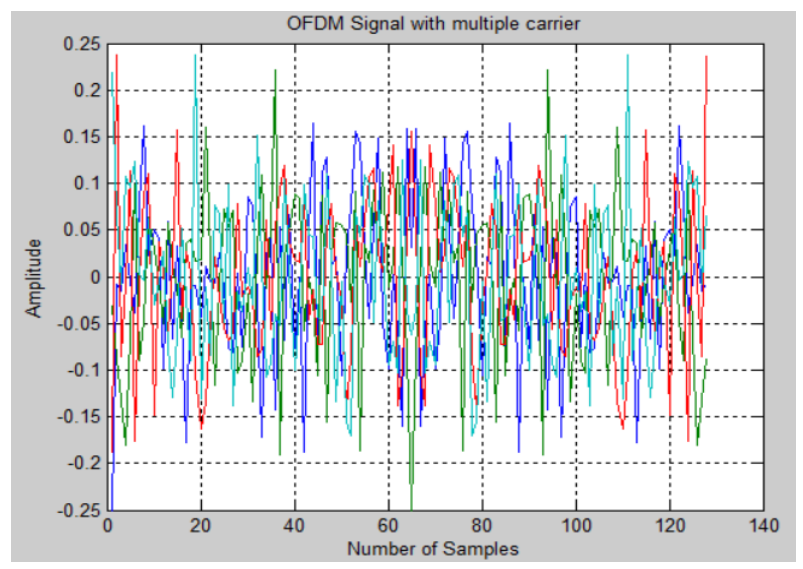

Fig.3 OFDM Signal with multicarrier 
To demonstrate proposed MIMO OFDM, Bit error rate is calculated as presented in figure (4)-(7) for BPSK, QPSK, 16QAM and 64-QAM modulation respectively.

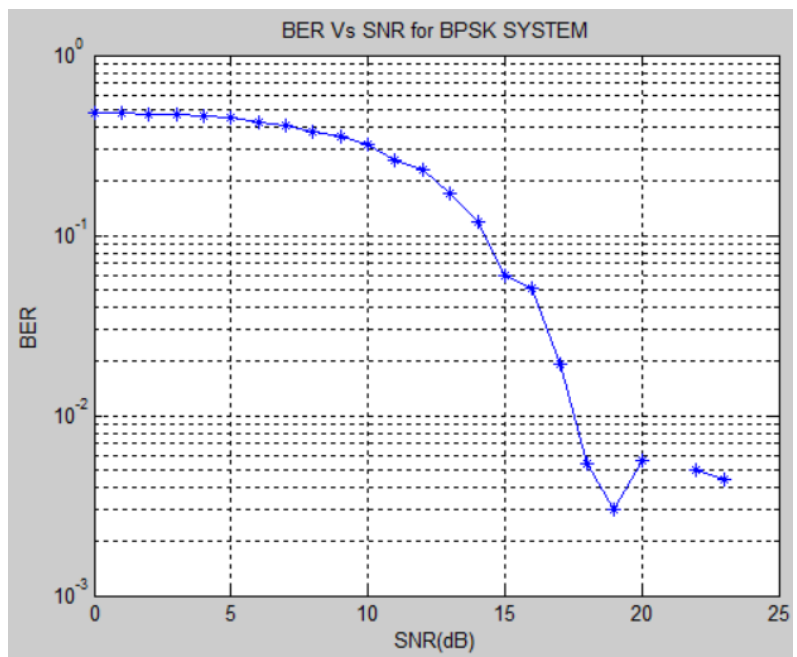

Fig.4 BER Vs. SNR for BPSK System

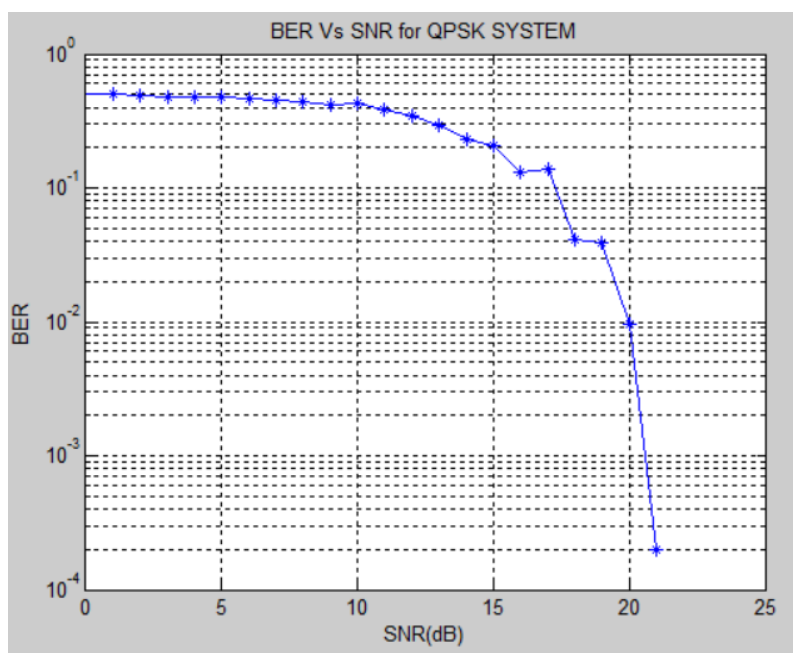

Fig.5 BER Vs. SNR for QPSK System

We further demonstrate this system for 16-QAM, 64-QAM, 256-QAM modulation scheme, as comparison of BER is presented in figure 8 .

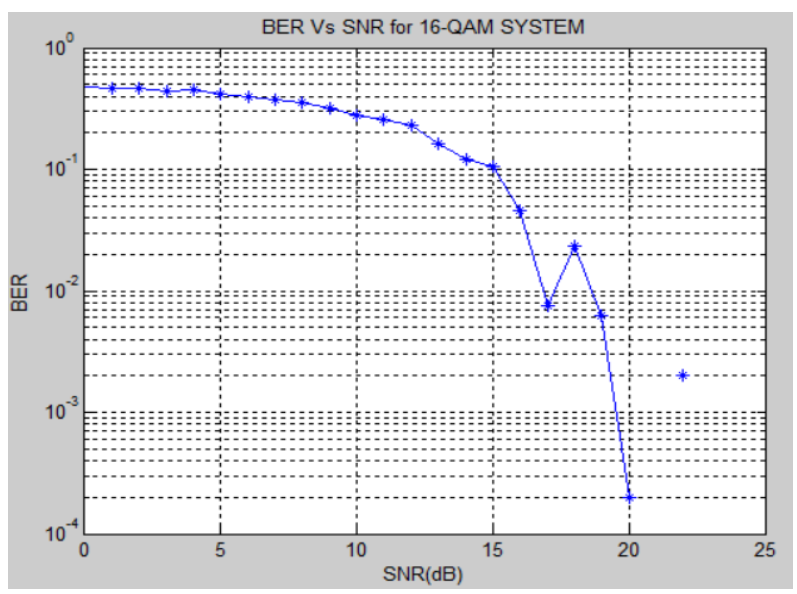

Fig.6 BER Vs. SNR for 16-QAM System

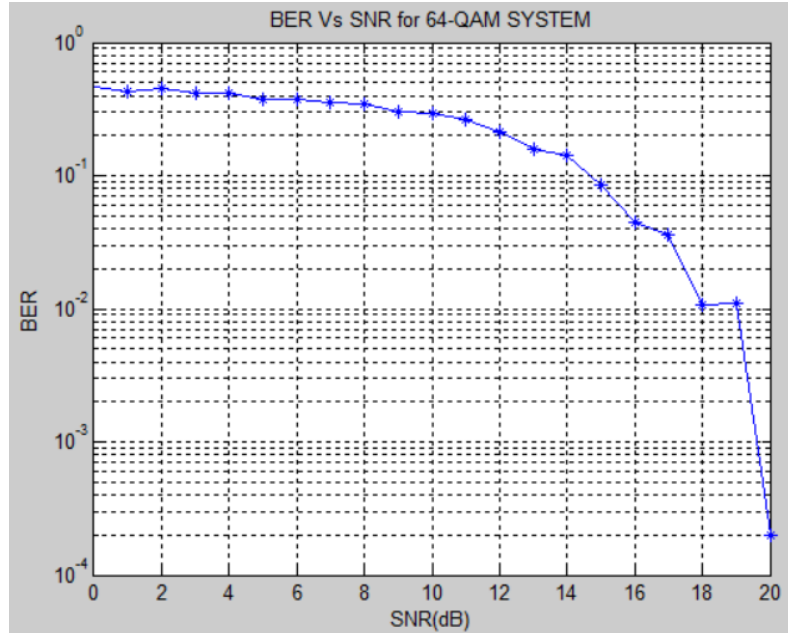

Fig.7 BER Vs. SNR for 64-QAM System

From figure (5), (6) and (7) it is observed that BER of 10-3 is achieved at $20 \mathrm{~dB}$ SNR under Rayleigh flat fading environment for QPSK, 16-QAM and 64-QAM respectively.

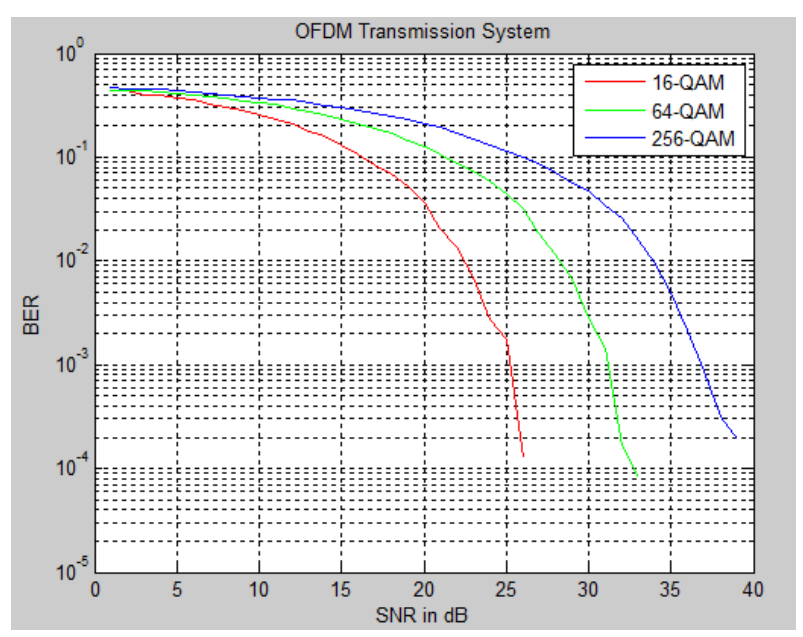

Fig.8 BER Vs. SNR for QPSK System

Figure 8 presents, comparison of BER for QAM modulation it is observed that, BER of $10-3$ is achieved at $25 \mathrm{~dB}, 30 \mathrm{~dB}$ and 35dB SNR under Rayleigh flat fading environment for 16QAM, 64-QAM and 256-QAM Respectively.

\section{CONCLUSION}

In proposed system results show the comparison analysis of BPSK, QPSK, 16-QAM,64-QAM and 256-QAM modulation schemes over AWGN channel. Based on the simulation results it is concluded that by using 16-QAM scheme better SNR performance for same value of BER is obtained as Compared to the BPSK and QPSK. Through simulation the BER performance of the system and the minimum required SNR to satisfy both high quality and low quality of data services is obtained.

\section{REFERENCES}

[1] Robert C. Daniels, Constantine M. Caramanis and Robert W. Heath, Members IEEE, "Adaptation in convolutionally coded MIMO-OFDM wireless systems through supervised learning and SNR ordering". IEEE Transactions on vehicular Technology, January, 2010, Vol. 59, No. 1. 
[2] R. Prasad, OFDM for wireless communication system, Artech House, 2004.

[3] Chan- Ho Choi and Gi-Hong, Sr. member IEEE, "Bit Interleaved Coded Transmission with Multilevel", IEEE GLOBE COM, 2009.

[4] Yi-Min Lin, Hsie-Chia Chang, and Chen-Yi Lee, "Improved High Code rate soft $\mathrm{BCH}$ decoder architectures with one extra error compensation", IEEE Transactions on very large scale integration(VLSI) systems, Nov 2013, Vol. 21, No. 11.

[5] G. Foschini and M. Gans, "On limits of wireless communications in a fading environment when using multiple antennas," wireless personal communications, March 1998, vol. 6, pp. 311-335.

[6] Jae-Min Kwak, Sung-Chul Lee, Ji-Woong Kim, Gi-Sik, Sung-Eon Cho and Hyo-Chang Pang, "The performance simulation of OFDM system using hierarchical 16 QAM on multipath fading channel", Journal of Security Engineering, Nov 2005, Vol. 2 No.1.

[7] K H H Wong, L. hanzo and R. Steele, "Channel coding for satellite mobile channels," Int. J satellite Communication, 1989, Vol, 7, pp. 143-163.

[8] L. Hanzo, K H H Wang, and R Steele, "Efficient Channel Coding and Interleaving schemes for mobile radio communications" in proc, Inst. Elec, Eng, Colloquium on Microcellular Mobile Radio, Feb, 22 1989, London, Savoy Place.

[9] Xiaojing Huang, "Effect of DC offset on OFDM system with zero padded suffix". IEEE, 2006.
[10] F. Rey, M. Lamarca and G. Vazquez, "Adaptive Interleaver based on rate- compatible punctured Convolutional codes", IEEE Transactions on communications, June 2009, Vol-57, No. 6.

[11] MATLAB, "HELP, Communication, toolbox, Block Interleaving".

[12] Vineet Chaturvedi, Vivek Kumar Gupta, DIT Dehradun, "Performance Analysis for Different Interleavers in Various Modulation Schemes with OFDM over an AWGN Channel", IOSR Journal of Engineers. April, 2012, Vol (2(4)) pp. 760-767.

[13] C N Chuah, DNC Tse, J M Khan, and R A Valenzuela, "Capacity scaling in MIMO wireless system under correlated fading, "IEEE Transactions on Information Theory, Vol. 48, No. 3, pp. 637-650, 2002.

[14] P F Driessen and G. J Foschini, "On the capacity formula for multiple input multiple output wireless channels: A Geometric Interpretation," IEEE Transactions Communications Feburary 1999. Vol. 2, pp. 173-176.

[15] Upena Dalal, Electronics Engineering Department,Sardar Vallabhbhai National Institute of Technology, Surat, "Wireless Communication", (C) Oxford University Press 2009.

[16] Z. Iqbal et al, "Analysis and Design of Coding and Interleaving in a MIMO OFDM Communication System," IEEE Transactions on Consumer Electronics, August 2012, Vol. 58, No. 3. 